\title{
Erratum to: Superior absorption and retention properties of foam-film silver dressing versus other commercially available silver dressing
}

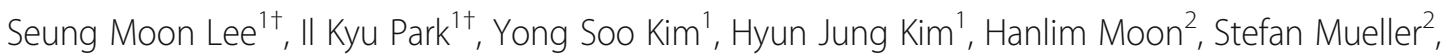
Harsha Arumugam ${ }^{2}$ and Young-IL Jeong ${ }^{3^{*}}$

\section{Erratum}

The original article contains an error [1] in the Discussion section, in the following sentence:

"[...] highly absorptive and moisture retentive dressings such as BETAplast, are a welcomed addition to wound management armamentarium."

The mention of 'BETAplast' in this sentence should instead be 'Medifoam Silver'.

\footnotetext{
Author details

${ }^{1}$ Genewel Co. Ltd, Gyeonggi-do, Korea. ${ }^{2}$ Mundipharma Pte. Ltd, Singapore, Singapore. ${ }^{3}$ Biomedical Research Institute, Pusan National University Hospital, 179 Gudeok-ro, Seo-gu, Busan 602-739, Republic of Korea.
}

Received: 15 November 2016 Accepted: 18 November 2016 Published online: 05 December 2016

\section{References}

1. Lee SM, et al. Superior absorption and retention properties of foam-film silver dressing versus other commercially available silver dressing. Biomater Res. 2016:20:22. doi:10.1186/s40824-016-0069-z.

\footnotetext{
* Correspondence: nanomed@naver.com

${ }^{\dagger}$ Equal contributors

${ }^{3}$ Biomedical Research Institute, Pusan National University Hospital, 179

Gudeok-ro, Seo-gu, Busan 602-739, Republic of Korea
} 